\title{
INFLUÊNCIA DE FATORES CLIMÁTICOS NO PANORAMA DA DENGUE NO BRASIL NO PERÍODO 2018-2019
}

\author{
INFLUENCE OF CLIMATE FACTORS ON THE DENGUE PANORAMA IN \\ BRAZIL IN THE 2018-2019 \\ Charlene Benício Farias Dias ${ }^{1}$ \\ Valentim Silva Monteiro ${ }^{2}$ \\ Vanessa Helena Pires da Costa do Nascimento ${ }^{3}$ \\ Maysa de Vasconcelos Brito ${ }^{4 *}$
}

RESUMO: A dengue é uma arbovirose de importância nacional devido o Brasil possuir as características climáticas que contribuem para a proliferação do vetor transmissor da doença. O objetivo do trabalho é apresentar o panorama da dengue no Brasil nos anos de 2018 e 2019 . Trata-se de um estudo de delineamento transversal longitudinal e retrospectivo de abrangência dos casos de dengue no Brasil nos anos de 2018 e 2019 segundo as regiões brasileiras, as unidadesfederativas, temperatura, precipitação, faixa etária e sexo, com foco, principalmente, na influência climática. Os dados para a pesquisa foram obtidos por meio do SINAN Net (casos de dengue); INMET (precipitação e temperatura média) e IBGE (população). Além disso foi realizado a correlação de Pearson com as variáveis temperatura e precipitação. Os resultados mostraram que no ano de 2018 houve 265.458 casos prováveis de dengue no país. Com concentração, principalmente na região centro-oeste, sudeste e nordeste. Em 2019, houve 1.558 .467 casos prováveis de dengue, aumento significativo. No entanto, a região com maior número de casos foi Sudeste, seguido da região Centro-Oeste e Nordeste. Ao realizar a correlação de Pearson nos municípios com maior incidência em 2018 e 2019, verificou-se quea pluviosidade e temperatura apresentaram uma correlação de fraca a forte, sendo que o fator temperatura apresentou correlação positiva apenas para Rio Branco, enquanto a precipitação apresentou maior grau de influência nas capitais estudadas. Dessa forma, com a pesquisa foi possível verificar a influência dos fatores ambientais, principalmente, temperatura e precipitação, na progressão dos casos de dengue no país.

Palavras-chave: Dengue. Epidemiologia. Fatores ambientais.

ABSTRACT: Dengue is an arbovirus of national importance because Brazil has the climatic characteristics that contribute to the proliferation of the vector that transmits the disease. The objective of thework is to present the panorama of dengue in Brazil in the years 2018 and 2019. This is a longitudinal and retrospective cross-sectional study of the scope of dengue cases in Brazil in the years 2018 and 2019 according to Brazilian regions, the

\footnotetext{
${ }^{1}$ ORCID: https://orcid.org/oooo-ooo3-1815-1117. Acadêmica de Biomedicina da Faculdade Estácio de Macapá, Brasil. E-mail: charlenebenicio@gmail.com.

${ }^{2}$ ORCID: https://orcid.org/oooo-ooor-5033-2140. Acadêmico de Biomedicina da Faculdade Estácio de Macapá, Brasil. E-mail: valentimmonteiro3@gmail.com.

3 ORCID: https://orcid.org/oooo-ooor-6662-7672. Acadêmica de Biomedicina da Faculdade Estácio de Macapá, Brasil. E-mail: vanessa.helena97@hotmail.com.

4 ORCID: https://orcid.org/oooo-ooor-6078-9996. Doutora em Doenças Tropicais (UFPA), Mestre em Neurociências e Biologia Celular (UFPA), Bacharel em Biomedicina (UFPA), Docente da Faculdade Estácio de Macapá, Brasil.E-mail: maysavb@yahoo.com.br.* Autora de correspondência.
} 
federative units, temperature, precipitation, age and sex, focusing mainly on the climatic influence. The data for the researchwere obtained through SINAN Net (dengue cases); INMET (precipitation and average temperature) and IBGE (population). In addition, Pearson's correlation was performed with thevariables temperature and precipitation. The results showed that in 2018 there were 265,458 probable dengue cases in the country. With concentration, mainly in the central-west, southeastand northeast regions. In 2019, there were $1,558,467$ probable dengue cases, a significant increase. However, the region with the highest number of cases was the Southeast, followed bythe Midwest and Northeast. When performing Pearson's correlation in the municipalities with the highest incidence in 2018 and 2019, it was found that rainfall and temperature showed a weak to strong correlation, with the temperature factor showing a positive correlation only for Rio Branco, while precipitation presented a greater degree of influence in the studied capitals. Thus, with the research it was possible to verify the influence of environmental factors, especially temperature and precipitation, on the progression of dengue cases in the country.

Keywords: Dengue. Epidemiology. Environmental factors.

\section{INTRODUÇÃO}

Dengue é uma síndrome febril de caráter agudo causada pelo vírus da dengue, além disso, é uma das arboviroses mais importantes que afetam o ser humano, visto a frequência, expansãoglobal e morbimortalidade associadas (SÁ ROCHA et al, 20II). É uma arbovirose que está presente em todo território nacional, sendo uma das doençasmais frequentes no Brasil. Isso ocorre porque o país atende as características que propicia a proliferação do vetor transmissor da doença. (SOUZA et al, 2019).

O vírus da dengue, pertencente à família Flaviviridae, é uma partícula esférica (5onm de diâmetro), onde contém cópias de três proteínas estruturais, uma bicamada de membrana derivada do hospedeiro e uma cópia do genoma de RNA de cadeia simples de sentido positivo(PERERA; KUHN, 2008; WHO, 2009; KHETARPAL; KHANNA, 2016). No Brasil, foi identificado apenas os sorotipos DENV-I ao DENV-4, com ordem de maior a menor virulência seguida porDENV-3, DENV-2, DENV-4 E DENV-I (FURTADO et al, 2019).

A primeira epidemia de dengue com base em confirmação laboratorial foi em Boa Vista,capital de Roraima, na década de 8o, que devido ao restrito trânsito de mercadorias e pessoas no período a doença não se disseminou para outros lugares. Todavia, em 1986, o vírus denguer emergiu no Rio de Janeiro e disseminou para o resto do país. Em relação ao vírus 2 e 3, estesforam introduzidos no país, também no Rio de Janeiro, em 1990 e 2002, respectivamente. E emzoro o vírus dengue 4 começou a circular no Brasil, identificado no Amazonas e no interior deSão Paulo (SEGURADO; CASSENOTE ; LUNA, 2016).

Historicamente, o vírus Dengue permanece em franco processo evolutivo, com situações de emergência e reemergência, se diferenciando do Dengue de cem anos atrás (CATÃO, 2012). O acompanhamento epidemiológico desta importante patologia, se faz necessário para propiciar um adequado planejamento de ações de controle e prevenção.

O curso epidemiológico da dengue e da dengue hemorrágica, sua forma mais grave, inclui a tríade de interação ecológica: hospedeiros humanos, um ou mais sorotipos do vírus dengue e mosquitos vetores (Aedes albopictus e Aedes aegypti). Os surtos de dengue são qualitativamente conhecidos por serem fortemente influenciados pela temperatura, umidade, precipitação e fatores socioeconômicos como a urbanização (XU et al, 2017). 
Poucos estudos detiveram-se a estudar quantitativamente a natureza dos efeitos ambientais na dinâmica da dengue no Brasil. Dessa forma, este trabalho teve como objetivo investigar a influência de fatores climáticos no panorama da dengue no Brasil nos anos de 2018e 2019 .

\section{METODOLOGIA}

O presente artigo traz um estudo de delineamento ecológico transversal longitudinal e retrospectivo de abrangência dos casos de dengue no Brasil nos anos de 2018 e 2019 segundo as regiões brasileiras, as unidades federativas, temperatura, precipitação, faixa etária e sexo, com o foco, principalmente, na influência climática. Segundo Bordalo (2006) esse tipo de pesquisa é dinâmico, pois investiga a causa da doença em grupos de casos novos a qual pode oscilar ao decorrer do tempo e em diferentes populações.

A pesquisa foi conduzida a partir de dados secundários obtidos por meio de consulta aoSistema de Informação de Agravos de Notificação - Sinan Net. As informações foram obtidas por meio de consulta eletrônica ao site DataSUS. Com os dados de casos de dengue coletados foi calculado a incidência de dengue em cada estado por Ioo.ooo habitantes de acordo com a população obtida pelo Instituto Brasileiro de Geografia e Estatística - IBGE -, com objetivo deverificar os estados com maior incidência de dengue nos dois anos de estudo. Em relação aos determinantes temperaturas e precipitação, os dados foram obtidos por meio do Instituto Nacional de Meteorologia - INMET.

Os dados foram tabulados utilizando o software Excel $2016 \mathrm{com}$ os resultados expressosem gráficos e/ou tabelas. Para a análise das variáveis das capitais dos estados com maior incidência foi realizado a correlação de Pearson entre as variáveis pluviosidade e temperatura média mensal. A correlação de Pearson é uma medida de variância entres duas variáveis, possuindo valores de $\mathrm{r}=-\mathrm{I}$ a I, sendo que o sinal indica se há uma relação positiva ou negativados dois fatores e o valor indica a intensidade entre eles. A correlação perfeita, I ou I, indica que o valor de uma variável segue o mesmo padrão da outra variável, ou seja, quanto mais pertode $\mathrm{I}$, independente do sinal, maior é a relação de dependência entre elas, no entanto, uma correlação com valor zero indica que não há relação existente (FIGUEIREDO, 2010).

Para saber se as correlações feitas durante a pesquisa eram fracas, moderadas ou fortes, utilizou-se da classificação de Cohen (1988), onde valores entre o, ıo e 0,29 podem ser considerados pequenos; entre 0,30 e 0,49 médios; e entre 0,50 e I grandes.

\section{RESULTADOS E DISCUSSÕES}

Inicialmente a dengue caracterizou-se como uma doença urbana por atingir os grandes centros populacionais - primeiro Sudeste e Nordeste, depois Norte e Centro-Oeste. Posteriormente a distribuição expandiu e observou-se epidemias em cidades de médio de pequeno porte. Sobre os picos epidêmicos, ocorreram três no país, em 2010, 2013 e 2015 commais de um milhão de casos prováveis notificados (SEGURADO; CASSENOTE; LUNA, 2016).

Entre 2003 a maio de 2019 foram registrados II.137.664 casos prováveis de dengue no Brasil, esses casos estão distribuídos em todo o país, com os maiores números de casos presentes nos municípios das regiões Centro-oeste e Sudeste, e com menor incidência na regiãoSul (SOUZA et al, 2019). 
Desse modo, caracterizamos a dinâmica de ocorrência dos casos de dengue no Brasil nos anos de 2018 e 2019, realizando a distribuição dos casos por mês de notificação. Observouse que no ano de 2018 houve 265.458 casos prováveis de dengue no país. Em relação a 2019, houve 1.558 .467 casos prováveis de dengue, um aumento significativo que corresponde a aproximadamente a 6 vezes na quantidade total de casos em comparativo ao ano de 2018 . Essadiferença no número de casos de dengue pode ser explicada pela re-emergência do sorotipo 2,devido à resistência da população sobre o sorotipo I (CARRERA, 2019).

Como é possível observar na Figura I, correspondente ao ano de 2018 e 2019, observase que o primeiro semestre do ano é o período que concentra o maior número de casos prováveis, com pico em abril de 2018 e em maio de 2019. Além disso, observa-se que em ambos os anos a partir de junho até setembro estabeleceu-se um período de remissão epidêmica, seguido de aumento gradativo em outubro em diante.

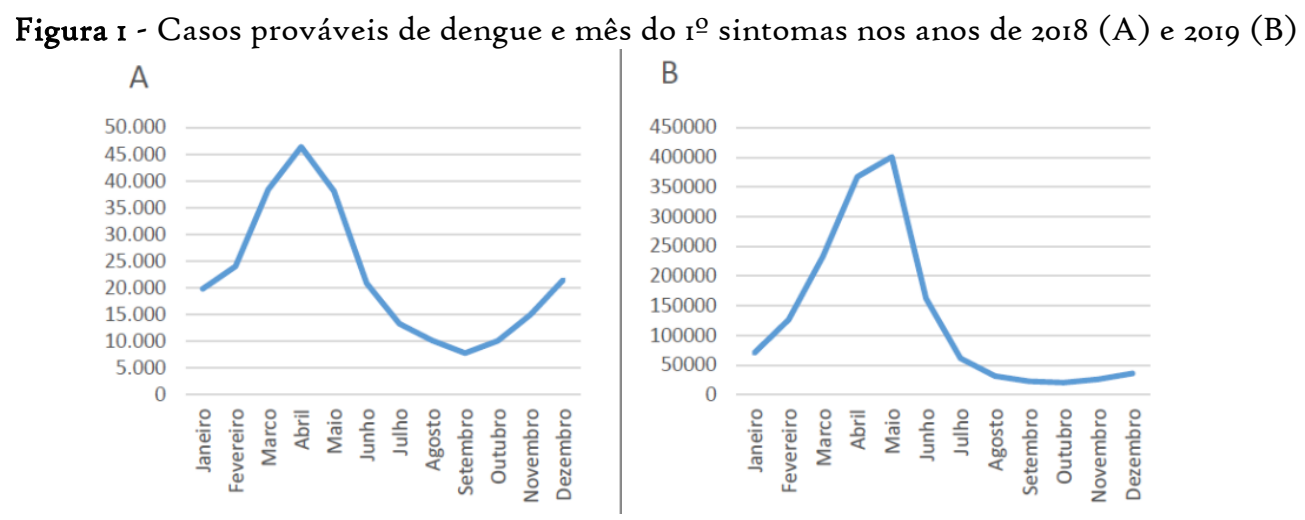

Fonte: Gráfico elaborado pelos autores de acordo com as informações obtidas pelo Ministério da Saúde/SVS -Sistema de Informação de Agravos de Notificação - Sinan Net

Essa apresentação na elevação do número de casos de dengue no país coincide commeses de maior chuva ao longo do ano. É bem conhecido que a temperatura, umidade relativa do ar e pluviosidade influenciam diretamente na dinâmica do vetor e nos picos de epidemia dadengue no Brasil, sendo que os agravos no primeiro semestre de cada ano estão relacionados ao aumento dos índices pluviométricos e variações de temperatura, que facilitam o aumento nonúmero de criadouros (VIANA; IGNOTTI, 2013).

Essa dinâmica parece ter sido mantida no período em estudo. Segundo registros do Instituto Nacional de Pesquisas Espaciais - INPE (2020) nos anos de 2018 e 2019, nos meses de janeiro, fevereiro, março, abril, outubro, novembro e dezembro concentram os maiores índices de precipitação. Nosdemais meses observa-se uma precipitação baixa ou inexistente. Assim, quando comparamos as observações da figura I com as precipitaçõestotais registradas em 2018 e 2019 pode-se identificar que os períodos de pico epidêmicos da Dengue foram acompanhados de maior precipitação e os de remissão coincidem com menor quantidade de precipitação.

O território brasileiro apresenta variação nas características climáticas, cobertura vegetal e meteorológica entre as diferentes regiões geográficas brasileiras. Dessa forma, para melhor investigar a influência de fatores ambientais com a ocorrência de dengue no país, procedeu-se previamente o recorte dos casos prováveis de dengue por região geográfica e mêsde ocorrência.

No ano de 2018 os casos se concentraram principalmente na região centro-oeste com I06.917 casos (40\%), seguido da região sudeste com 72.620 (27\%), região nordeste com 66.5 II 
casos (25\%), região norte com 17.675 (7\%) e por fim a região sul com 1.737 casos prováveis (I\%). Em relação a 2019, no entanto, a região que concentrou o maior número de casos foi sudeste com 1.026.537 (66\%), seguido da região Centro-Oeste com 228.123 casos (I5\%), região Nordeste com 216.86o (I4\%), região Sul com 49.865 casos (3\%) e por fim região Nortecom 37.082 casos $(2 \%)$. Esses dados mostram que existem diferenças nas características regionais brasileiras ao longo do tempo. Câmara et al. (2007) analisando a série histórica de 1986-2003 para cada região brasileira, identificaram maior concentração das notificações nas regiões Nordeste e Sudeste do país, correspondendo juntas a $86 \%$ do total de casos, enquanto Centro-Oeste registrou apenas $7,6 \%$ no período.

Nesses dois anos, conforme pode ser visto na Figura 2, observa-se que semelhante em todas as regiões brasileiras, o primeiro semestre do ano é o período que concentra o maior número de casos prováveis de dengue.

Figura 2 - Casos prováveis de dengue por região de notificação e mês do Io sintomas nos anos de 2018

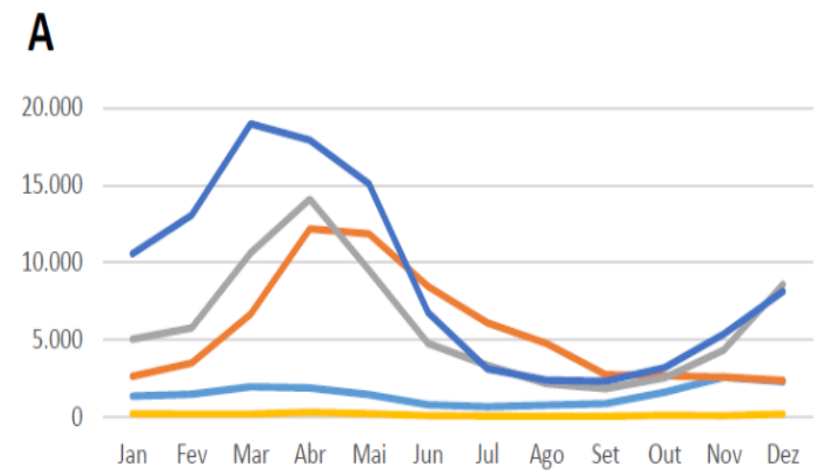

(A) e 2019 (B).

Fonte: Gráfico elaborado pelos autores de acordo com as informações obtidas pelo Ministério da

B

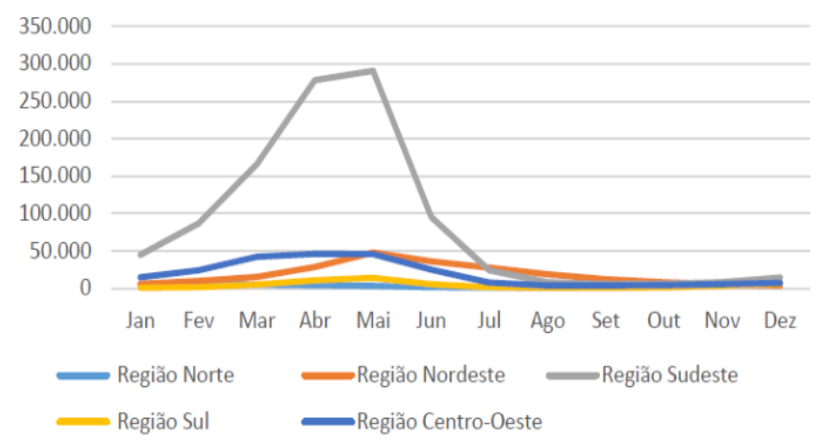

Saúde/SVS - Sistema de Informação de Agravos de Notificação - Sinan Net

A fim de explorar a relação causal de fatores climáticos com incidência de dengue nos principais recantos epidêmicos e urbanos do país no período em estudo, procedeu-se a análise de correlação de Pearson da variável incidência de dengue com as variáveis temperatura e precipitação para as capitais dos estados que apresentaram as maiores incidência em 2018 e 2019. Assim, avaliou-se a distribuição dos casos de dengue por unidade federativa (Figura 3).Em 2018, os estados com maior incidência foram Goiás, Acre e Rio Grande do Norte. No ano de 2019, os estados que apresentaram as maiores incidência de dengue foram Minas Gerais, Mato Grosso do Sul e Goiás. 
Figura 3 - Incidência de dengue em cada estado por Ioo.ooo habitantes no ano de 2018 (A) e 2019 (B)

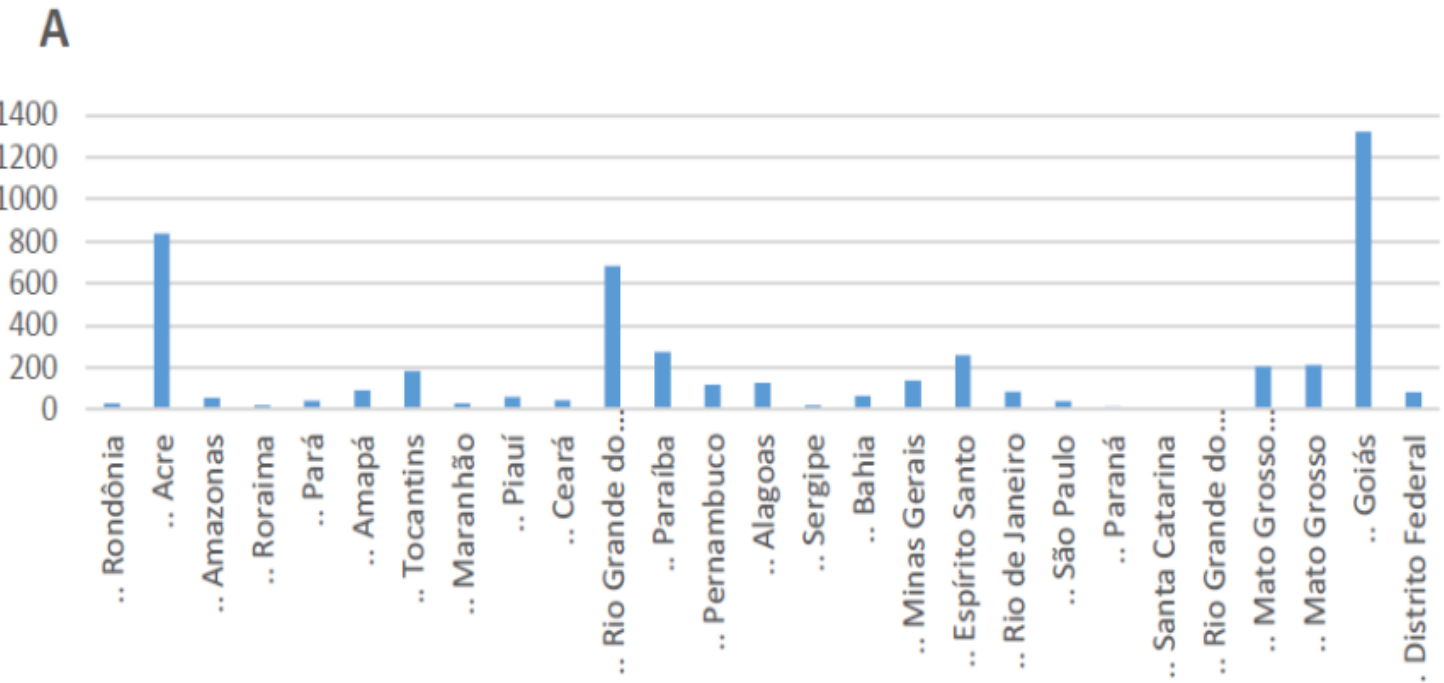

\section{B}

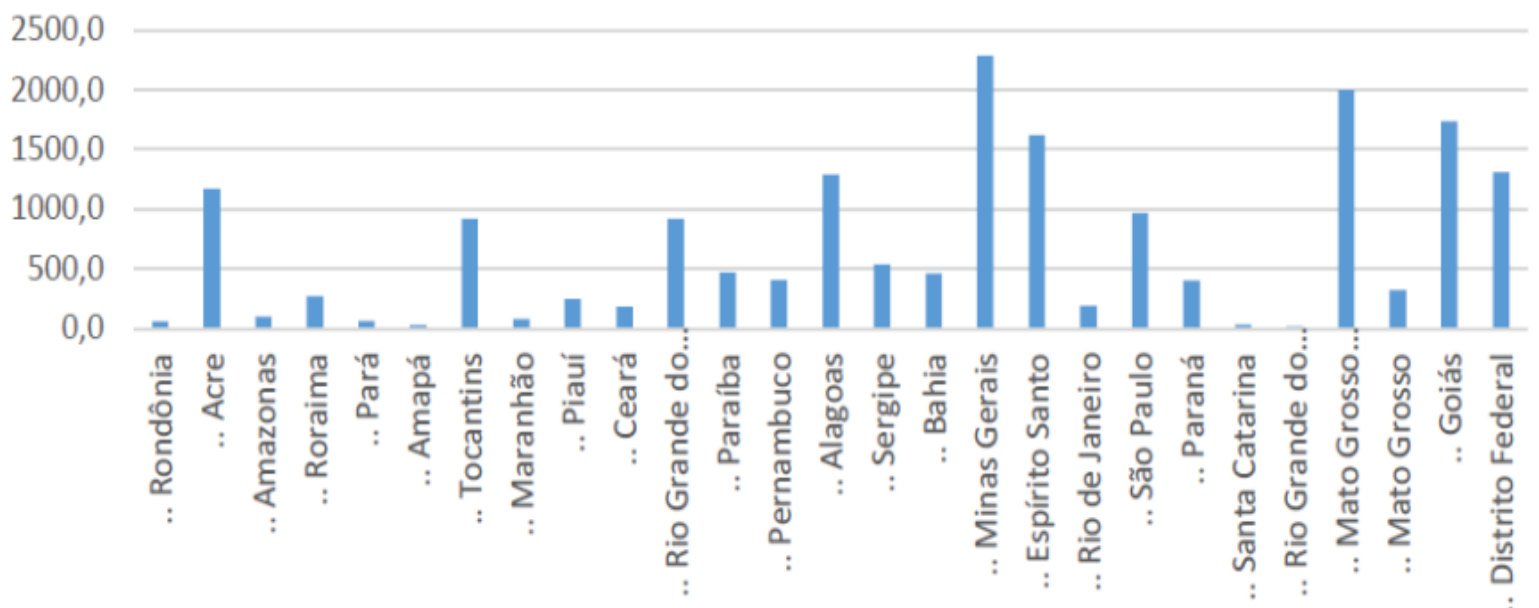

Fonte: Gráfico elaborado pelos autores de acordo com as informações obtidas pelo Ministério daSaúde/SVS - Sistema de Informação de Agravos de Notificação - Sinan Net

No caso de Goiânia, capital de Goiás, observa-se na Figura 4 que os meses fevereiro, março e abril concentraram as maiores incidências de dengue em 2018, correspondendo também aos meses de maior pluviometria. É possível notar certa semelhança entre os meses demaior chuva com os meses de maior incidência de dengue, no entanto, ao ser realizado a correlação de Pearson, verificou-se que essas duas variáveis apresentaram uma correlação positiva mediana $(r=0,4)$. Sobre a relação entre a temperatura mensal média e a incidência, a correlação se apresentou fraca e de forma negativa $(r=-0, \mathrm{I})$. 
Figura 4 -Incidência mensal de dengue, Precipitação pluviométrica mensal e temperatura média mensalno município de Goiânia - GO - no período de 2018.

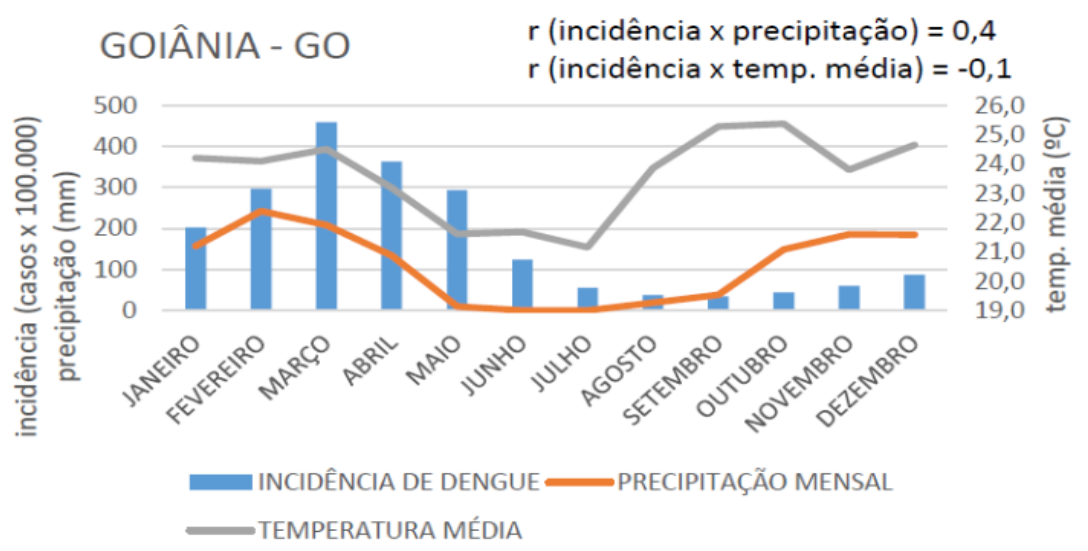

Fonte: Gráfico elaborado pelos autores de acordo com as informações obtidas pelo Ministério da Saúde/SVS - Sistema de Informação de Agravos de Notificação - Sinan Net -, dados da população doIBGE e pluviosidade e temperatura média do INMET.

Em relação ao Rio Branco (Figura 5), capital do Acre, a correlação de Pearson foi mediana tanto para a variáveis incidência $x$ precipitação $(r=0,3)$ quanto para incidência $x$ temperatura média mensal $(r=0,4)$. Os meses que tiverem a incidência mais alta foram outubro, novembro e dezembro, e os meses com maior pluviometria, fevereiro, abril e dezembro.

Figura 5-Incidência mensal de dengue, Precipitação pluviométrica mensal e temperatura média mensalno município de Rio Branco - AC - no período de 2018.

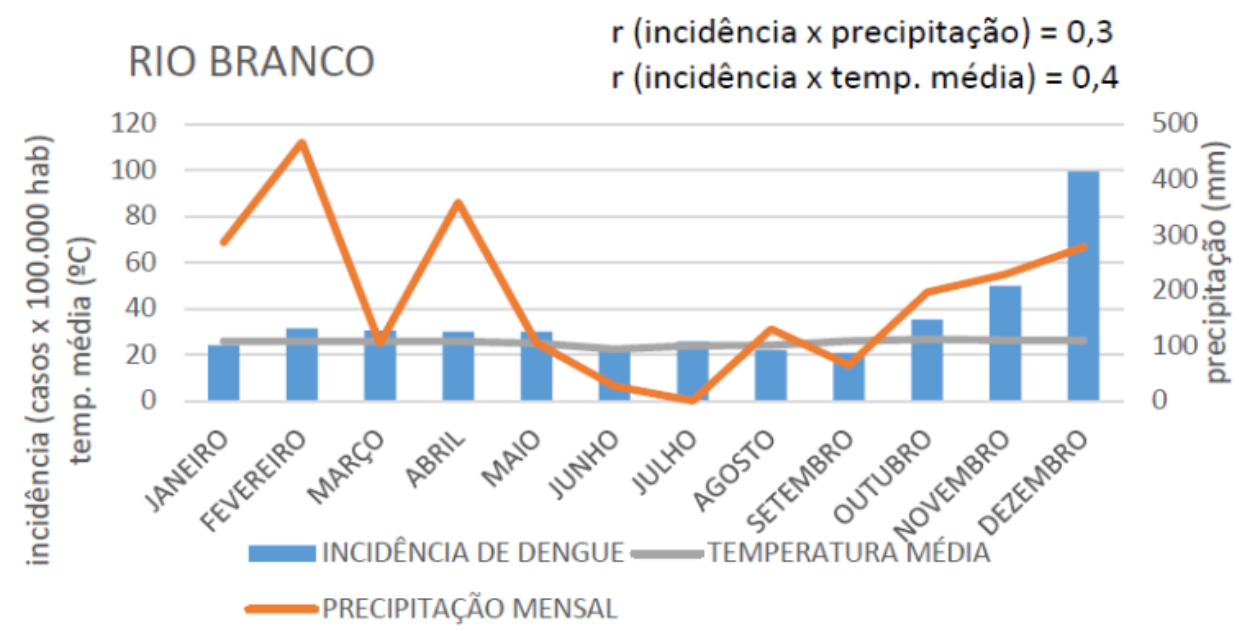

Fonte: Gráfico elaborado pelos autores de acordo com as informações obtidas pelo Ministério da Saúde/SVS

- Sistema de Informação de Agravos de Notificação - Sinan Net -, dados da população doIBGE e pluviosidade e temperatura média do INMET.

No caso da capital do Rio Grande do Norte, Natal (Figura 6), houve uma forte correlação positiva das variáveis incidência x precipitação $(r=0,8)$. Sendo os meses de abril, maio e junhocom os maiores índices de incidência de casos e uns dos meses com maiores valores de precipitação. 
Tratando-se das variáveis incidência e temperatura média mensal, estas apresentaram uma correlação negativa mediana $(r=-0,4)$.

Figura 6 -Incidência mensal de dengue, Precipitação pluviométrica mensal e temperatura média mensalno município de $\mathrm{Natal}$ - RN - no período de 2018.
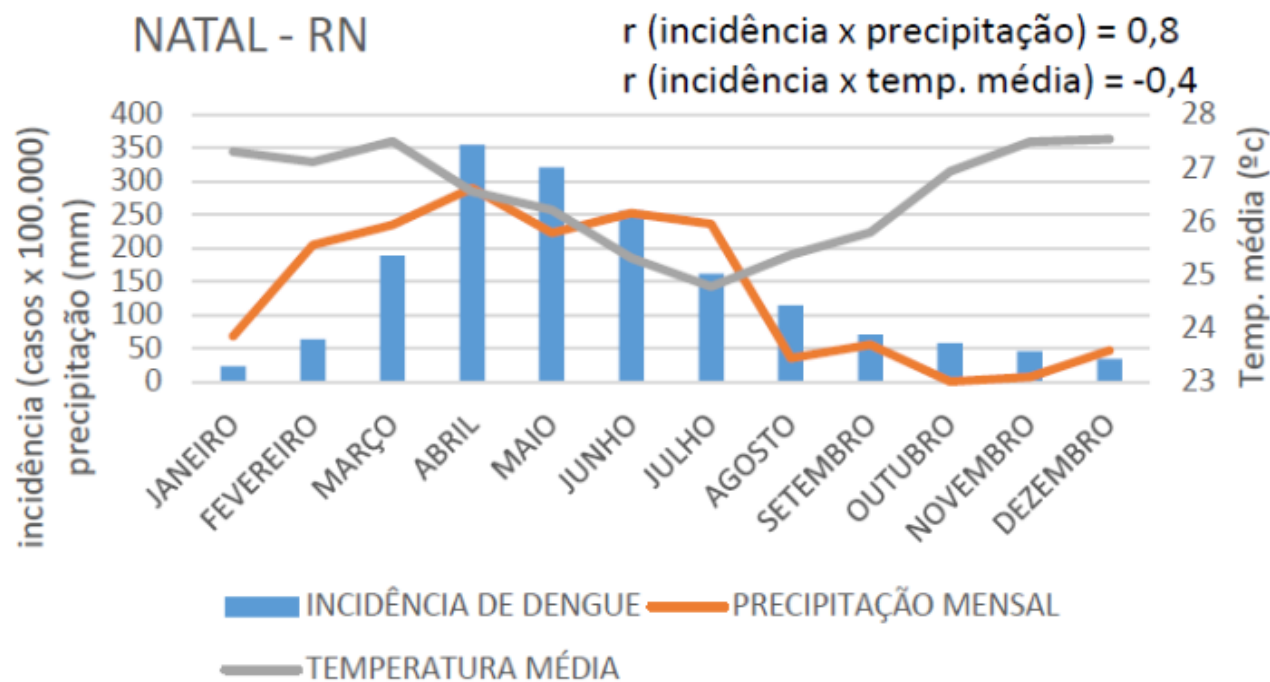

Fonte: Gráfico elaborado pelos autores de acordo com as informações obtidas pelo Ministério da Saúde/SVS

- Sistema de Informação de Agravos de Notificação - Sinan Net -, dados da população doIBGE e pluviosidade e temperatura média do INMET.

Em 2019, o estado com maior incidência foi Minas Gerais, e a correlação de pearson nacapital Belo Horizonte apresentou-se negativa e fraca tanto para as variáveis precipitação $(\mathrm{r}=-0, \mathrm{I})$ quanto temperatura média $(\mathrm{r}=-0, \mathrm{I})$. No caso de Campo grande, capital do Mato Grosso Sul, e Goiânia, capital de Goiás, a correlação entre incidência de casos e precipitação foram positivas e medianas ( $r=0,4$ e $r=0,3$, respectivamente), entretanto para as variáveis incidênciade casos $\mathrm{x}$ temperatura média mensal, em Campo Grande a correlação foi negativa e fraca $(r=-0,1)$, e em Goiânia foi negativa mediana $(r=-0,3)$ (Figura 7$)$.

Figura 7 -Incidência mens
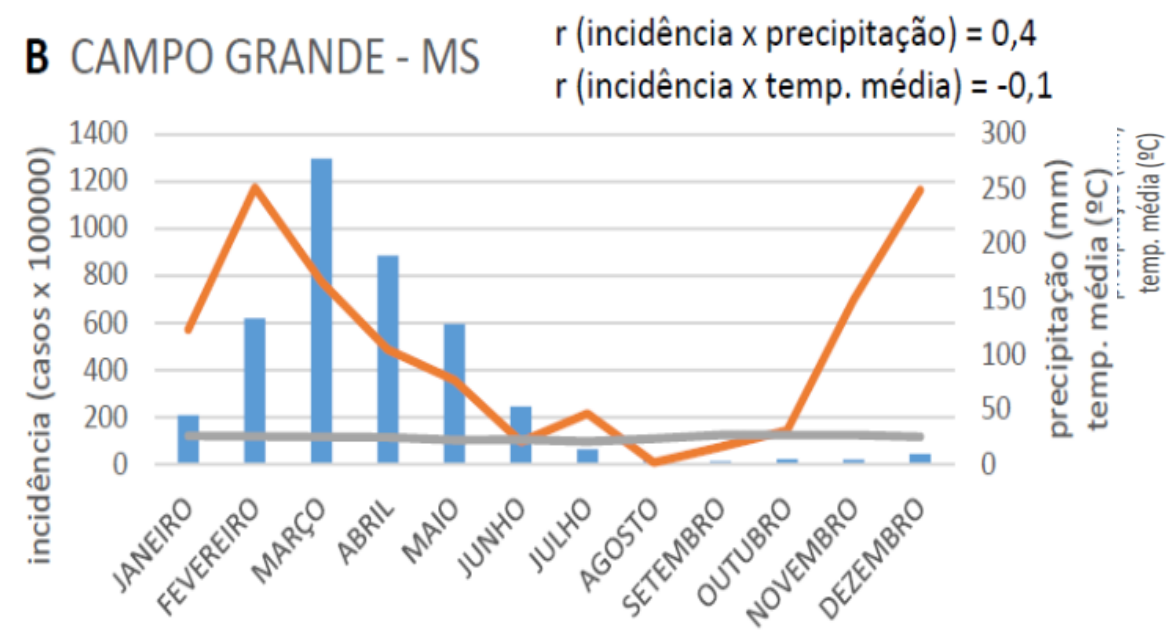


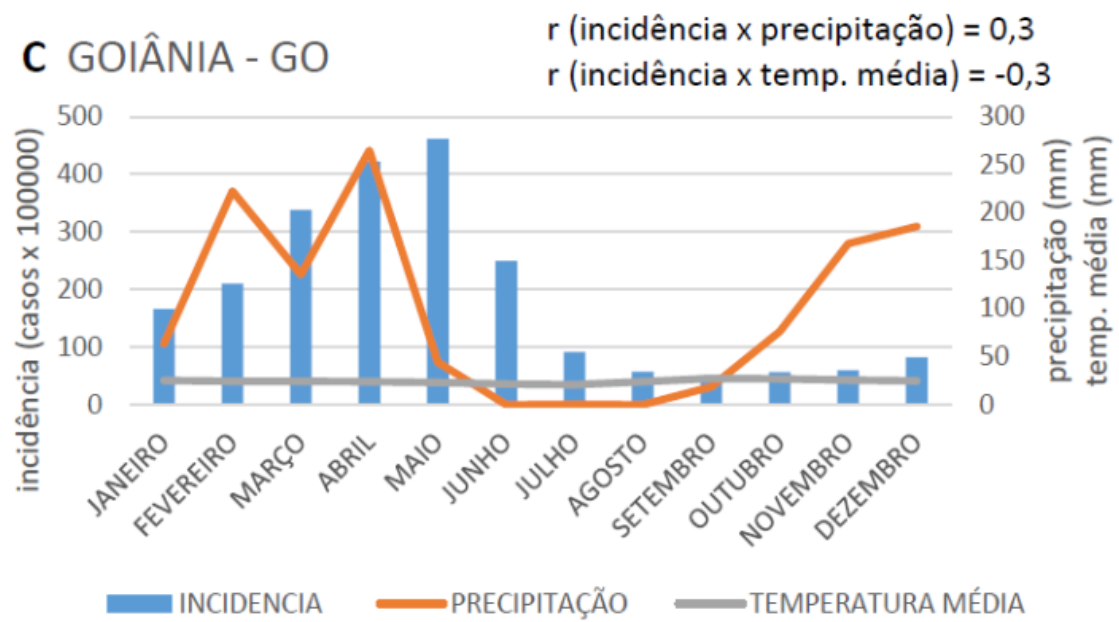

Fonte: Gráfico elaborado pelos autores de acordo com as informações obtidas pelo Ministério da Saúde/SVS Sistema de Informação de Agravos de Notificação - Sinan Net -, dados da população doIBGE e pluviosidade e temperatura média do INMET

Nota-se que apesar dos gráficos apresentaram uma certa correspondência em relação aocomportamento das variáveis, a correlação pode não ser tão significativa, visto que no caso dadengue, há outros fatores determinantes para a propagação da doença.

A pesquisa desenvolvida por Barbosa e Silva (2015) apresentou uma correlação fraca em relação a pluviosidade na cidade de $\mathrm{Natal}$ no período de 2008 e 2012. Entretanto, no trabalho de Teixeira e Cruz (20II) na cidade de Rio de Janeiro, a correlação da variável índice pluviométrico apresentou-se positiva, assim como para as demais variáveis estudadas (índice de Breteau, índice de Gini e índice de desenvolvimento social), com isso os pesquisadores constataram a significância das variáveis socioambientais no processo de transmissão de dengue.

Scandar el al (2010) realizaram uma análise em São José do Rio Preto (SP) entre 1990 e 2005, com as variáveis entomológicas, ambientais e socioeconômicas. Assim, no período de chuva verificaram que o índice larvário crescia, mas que esse crescimento dependia da disponibilidade dos criadouros. Observa-se então, conforme mencionando por Barbosa e Silva(2015), que é necessário uma investigação mais individualizada sobre cada um dos fatores: densidade populacional, taxa de crescimento, saneamento, temperatura, chuva, saneamento, assim como outras variáveis relacionadas aos aspectos socioambientais e demográficos, para poder entender a dinâmica espacial de dengue, visto também que depende da realidade de cada município.

Segurado, Cassenote e Luna (2016), mencionam que o processo de urbanização das cidades em rápida transformação promove mudanças nas condições de saúde da população comefeitos antagônicos. Este pode ser um fator relevante para a manutenção ou controle de doençascomo a dengue. Dessa forma, ao modo que esse processo possibilita maior facilidade de acessoa programas e serviços, por outro lado, impulsiona maior densidade demográfica e alterações tanto do ambiente físico quanto das relações sociais. Assim, pode haver modificação na ocorrência de diversos agravos à saúde devido fatores de natureza ambiental, demográfica, sociocultural e econômico.

Diante desse cenário nacional, um dos possíveis fatores que contribuíram para esse aumento foi o crescimento urbano desordenado, que gera como consequência aumento populacional, e em contrapartida mais resíduos sólidos são produzidos e descartados no 
ambiente, favorecendo o aumento na proliferação do Aedes aegypti. Além disso, a dengue tornou-se uma doença negligenciada pela sociedade por acreditarem que seja uma virose inofensiva. Além disso, o aumento no número de casos no ano 2019 também está relacionado a re-emergencia do sorotipo 2 , haja vista a imunidade adquirida do sorotipo $\mathrm{I}$.

\section{CONCLUSÃO}

O presente estudo possibilitou uma análise na dinâmica da incidência de casos de dengue no Brasil. Procurou-se fazer um levantamento de casos prováveis de dengue em nível regional, estadual e mensal, observando o período de maior incidência e ano. A partir das informações coletadas no site DataSUS, observou-se que em 2019 houve um aumento significativo de casos.

Os resultados mostraram que o fator temperatura apresentou correlação positiva apenaspara Rio Branco, enquanto precipitação apresentou maior grau de influência na incidência de dengue nas capitais estudadas, todavia essa apresentação não foi homogênea, indicando que cada espaço regional apresenta peculiaridades causais que devem ser consideradas na implementação de plano de controle para a dengue.

Ressalta-se assim a importância das análises dos fatores ambientais periodicamente, pois essa variação de interferência causal pode ser influenciada por alterações climáticas. Também se faz necessário o desenvolvimento de modelos matemáticos que possibilitem melhores projeções dos efeitos das mudanças climáticas na incidência da dengue.

É necessário salientar, que a progressão dos casos de dengue, conforme visto no decorrerda pesquisa, parte de fatores ambientais, no entanto, também devem ser avaliados em futuras pesquisas a influência das variáveis socioeconômicas na progressão da doença no país.

Nesse sentido, o acompanhamento epidemiológico dessa síndrome febril torna-se importante para o adequado planejamento de ações de controle e prevenção pelo poder público.Assim, também, as campanhas ao combate ao vetor devem ser intensificadas com o objetivo desensibilizar e conscientizar a população acerca da gravidade da doença, devido o vetor da dengue também ser transmissor de outras viroses.

Tendo em vista os aspectos observados, é necessário, nas escolas, a educação ambiental, como instrumento norteador a respeito dos problemas ambientais e sociais ocasionados pelo descarte incorreto de resíduos sólidos nas ruas e no ambiente em geral.

\section{REFERÊNCIAS BIBLIOGRAFICAS}

BARBOSA, Isabelle Ribeiro; DA SILVA, Lúcio Pereira. Influência dos determinantes sociais e ambientais na distribuição espacial da dengue no município de Natal-RN. Revista Ciência Plural, v. I, n. 3, p. 62-75, 2015.

Brasil (2020). Ministério da Saúde. Secretaria de Vigilância em Saúde. Casos de Dengue. Brasil. Disponível: http://tabnet.datasus.gov.br/cgi/deftohtm.exe?sinannet/cnv/denguebbr.def. Acessado 29 desetembro de 2020 .

BORDALO, Alípio Augusto. Estudo transversal e/ou longitudinal. Revista Paraense de 
Medicina, v. 20, n. 4, p. 5, 2006. Recuperado em 03 de dezembro de 2020

http://scielo.iec.gov.br/scielo.php?script=sci_arttext\&pid=SoI0159072006000400oor\&lng=p t\&tlng=pt.

CÂMARA, Fernando Portela et al. Regional and dynamics characteristics of dengue in Brazil: a retrospective study. Revista da Sociedade Brasileira de Medicina Tropical, v. 40, n. 2, p. 192-196, 2007.

CARRERA, M. (2019). Re-emergência do Sorotipo 2 do Vírus da Dengue no Brasil é um dos Responsáveis pelo Aumento de 60o\% dos Casos da Doença no País. Disponível em https://limhc.fm.usp.br/portal/entrada-de-novo-grupo-de-virus-da-dengue-no-brasil-eum-dos-responsaveis-pelo-aumento-de-6oo-dos-casos-da-doenca-no-pais/

CATÃO, R. D. C.. Dengue no Brasil: abordagem geográfica na escala nacional. Coleção PROPG Digital (UNESP), 2012.

COHEN, J., Statistical power analysis for the behavioral sciences. Hillsdale, NJ, Erlbaum, 1988.

FIGUEIREDO FILHO, Dalson Britto; SILVA JUNIOR, José Alexandre. Desvendando os Mistérios do Coeficiente de Correlação de Pearson (r). Revista Política Hoje, v. I8, n. I, p. II5-I46, 2010. Recuperado de https://periodicos.ufpe.br/revistas/politicahoje/article/view/3852/3156

FURTADO, A. N. R. et al. Dengue e seus avanços. Revista Brasileira de analyses clinicas, v. 5I, n. 3, p. I-5, 2019.

INMET - Instituto Nacional de Meteorologia. Dados Históricos: BDMEP - Banco de Dados Meteorológicos para Ensino e Pesquisa. Disponível em: http://www.inmet.gov.br/portal/index.php?r=bdmep/bdmep.

Acesso em 3 dez. 2020.

KHETARPAL, Niyati; KHANNA, Ira. Dengue fever: causes, complications, and vaccine strategies. Journal of immunology research, v. 2016, 2016.

PERERA, Rushika; KUHN, Richard J. Structural proteomics of dengue virus. Current opinion in microbiology, v. II, n. 4, p. 369-377, 2008.

SÁ ROCHA, Allesyo Patrick et al. Dengue: história natural e definição de casos graves e potencialmente graves. Rev Med Minas Gerais, v. 3116o, p. 370, 2011.

SCANDAR, Sirle Abdo Salluom et al. Dengue em São José do Rio Preto, Estado de São Paulo, Brasil, 2005: fatores entomológicos, ambientais e socioeconômicos. BEPA. Boletim Epidemiológico Paulista (Online), v. 7, n. 81, p. 04-16, 2010. 
SEGURADO, Aluisio Cotrim; CASSENOTE, Alex Jones; LUNA, Expedito de Albuquerque. Saúde nas metrópoles-Doenças infecciosas. Estudos avançados, v. 30, n. 86, p. 29-49, 2016.

SOUZA, A., LEITE, P., FREIRE, D. et al. Dengue. Boletim Epidemiológico. (Número especial: Vigilância em Saúde no Brasil 2003|2009: dacriação da Secretaria de Vigilância em Saúde aos dias atuais), 2019. Disponível em: http://www.saude.gov.br/ boletinsepidemiologicos.

SOUZA, Marcela Tavares de; SILVA, Michelly Dias da; CARVALHO, Rachel de. Revisão integrativa: o que é e como fazer. Einstein (São Paulo), v. 8, n. I, p. I02-106, 2010.

TEIXEIRA, Tatiana Rodrigues; CRUZ, Oswaldo Gonçalves. Spatial modeling of dengue and socio-environmental indicators in the city of Rio de Janeiro, Brazil Modelagem espacial da dengue e variáveis socioambientais no Município do. Cad. Saúde Pública, v. 27, n. 3, p. 591-602, 2011. https://dx.doi.org/ro.1590/Soro2-31IX2011000300or9

VIANA, Dione Viero; IGNOTTI, Eliane. A ocorrência da dengue e variações meteorológicas no Brasil: revisão sistemática. Revista Brasileira de Epidemiologia, v. ı6, p. 240-256, 2013.

WORLD HEALTH ORGANIZATION et al. Dengue: guidelines for diagnosis, treatment, prevention and control. World Health Organization, 2009.

XU, Lei et al. Climate variation drives dengue dynamics. Proceedings of the National Academy of Sciences, v. II4, n. I, p. II3-II8, 2017. https://doi.org/10.1073/pnas.1618558114 